\title{
Multi topological approximations of rough set theory
}

\author{
A.S. Salama* \\ Mathematics Department, \\ Faculty of Science, \\ Tanta University, \\ P.O. Box 20, Tanta, 31527, Egypt \\ Fax: 0020403404914 \\ and \\ Faculty of Science, Shaqra University, \\ P.O. Box 18, Al-Dawadmi, \\ 11911, Saudi Arabia \\ Fax: 0096616423918 \\ E-mail: dr_salama75@yahoo.com \\ *Corresponding author

\section{O.G. El-Barbary}

Statistics and Computer Science Department,

Faculty of Science,

Tanta University,

P.O. Box 20, Tanta, 31527, Egypt

Fax: 0096616423918

E-mail: omniaelbarbary@yahoo.com

\begin{abstract}
In this paper, we generalise Pawlak's approximation spaces to topological approximation spaces. These topological approximation spaces are generated using many topological notions such as regular open sets, semi-open sets, pre-open sets, $\gamma$-open sets, $\alpha$-open sets and $\beta$-open sets and others. The basic definitions and properties of these topological approximation spaces are introduced and sufficiently illustrated. We put all topological generalisations in one generalisation called $\mathrm{m}$-generalisation spaces.
\end{abstract}

Keywords: topological spaces; rough sets; rough approximations; information system; data reduction.

Reference to this paper should be made as follows: Salama, A.S. and El-Barbary, O.G. (2013) 'Multi topological approximations of rough set theory', Int. J. Granular Computing, Rough Sets and Intelligent Systems, Vol. 3, No. 1, pp.1-19.

Biographical notes: A.S. Salama received his BS in Mathematics from Tanta University, Tanta, Egypt in 1998 and MS in Topological Rough Sets from the University of Tanta in 2002. He worked at Tanta University from 1998 to 2008. He studied as a PhD student in topology and information systems at Tanta University, Tanta, Egypt from 2002 to 2004. Currently, he is an Assistant Professor of Mathematics and Computer Science at Shaqra University, KSA. His research interests include artificial intelligence, rough set, data mining, topology, fuzzy sets, and information systems. 
O.G. El-Barbary received her BSc in Statistics and Computer Science from Faculty of Science, Tanta University, Egypt, in 2004, MSc in Computer Science from Faculty of Science, Menofya University, Egypt, in 2007 and PhD in Computer Science from Menofya University in 2012. She is an Assistant Professor in Faculty of Science, Shaqra University. Her research interests include rough set, fuzzy set, information system, information retrieval, natural language processing and document processing.

\section{Introduction}

These days we use large amounts of data, which are stored in relational or latterly in object databases. These data have low information value. That is why we started to talk about the process of knowledge discovery in databases in the beginning of the 1990s. This process is divided into a number of consequent steps.

Rough set theory, proposed by Pawlak in the early 1980s (Pawlak, 1982), is an extension of set theory for the study of intelligent systems characterised by inexact, uncertain or insufficient information. Many proposals have been made for generalising and interpreting rough sets (Abu-Donia, 2008; Abu-Donia and Salama, 2008; Abu-Donia et al., 2007; Chuchro, 1993; Alpigini et al., 2002; Liu and Sai, 2009; Ouyang et al., 2010). Moreover, this theory may serve as a new mathematical tool to soft computing besides fuzzy set theory, and has been successfully applied in machine learning, information sciences, expert systems, data reduction, and so on. Recently, lots of researchers are interested to generalise this theory in many fields of applications (Bonikowski, 1994; Bryniaski, 1998; Cattaneo, 1998). In Pawlak's original rough set theory, partition or equivalence (indiscernibility) relation is an important and primitive concept. But, partition or equivalence relation is still restrictive for many applications. To study this issue, several interesting and meaningful generalisations to equivalence relation have been proposed in the past, such as tolerance relations, similarity relations, topological bases and sub-bases (Lashin et al., 2005) and others (Bonikowski et al., 1998; Kryszkiewicz, 1998). Particularly, some researchers have used coverings of the universe of discourse for establishing the generalised rough sets by coverings (Deng et al., 2007). Others (Banerjee and Pal, 1995; Biswas, 1992; Degang et al., 2008; Liu, 2008a) combined fuzzy sets with rough sets in a fruitful way by defining rough fuzzy sets and fuzzy rough sets. Furthermore, another group has characterised a measure of roughness of a fuzzy set making use of the concept of rough fuzzy sets (Biswas, 1994; Dubois and Prade, 1990). They also suggested some possible real world applications of these measures in pattern recognition and image analysis problems. Some results of these generalisations are obtained about rough sets and fuzzy sets in Chakrabarty et al. (2000), Chen and Li (2007), Gong et al. (2008), Li et al. (2008), Liu (2008b), Nakamura (1988) and Nanda and Majumda (1992). Rough set theory is a recent approach for reasoning about data. This theory depends basically on a certain topological structure and has achieved a great success in many fields of real life applications. The concept of a topological rough set is one of the most important topological generalisation of rough sets. In Abd El-Monsef et al. (1983), the concept of $\beta$-open sets is introduced and in Hatir 
and Noiri (2006) the concept of $\delta \beta$-open sets is introduced. This paper is organised as follows:

In Section 2, we give some of the topological notions that will generalise to rough sets. In Section 3, we discuss the fundamentals of Pawlak's rough set theory. Section 4 studies the generalisations of the topological notions to rough sets and introduce new rough approximations. Section 5 is devoted to introduce the theoretical results of the topological rough approximations. An application to rule induction and data mining in information systems using these approximations is introduced in Section 6. The proper conclusion is given in Section 7.

\section{Topological basic concepts}

A topological space (Banerjee and Pal, 1995) is a pair $(U, \tau)$ consisting of a set $U$ and family $\tau$ of subsets of $U$ satisfying the following conditions:

$1 \phi, U \in \tau$

$2 \tau$ is closed under arbitrary union

$3 \tau$ is closed under finite intersection.

The pair $(U, \tau)$ is called a topological space. The elements of $U$ are called points of the space and the subsets of $U$ belonging to $\tau$ are called the open sets. The complement of the open sets are called closed sets.

For a subset $A$ of a space $(U, \tau), \operatorname{cl}(A), \operatorname{int}(A)$ and $C(A)$ denote closure, interior and complement of $A$ in $X$ respectively.

A subset $A$ of a topological space $(U, \tau)$ is called:

1 semi-open set (Hatir and Noiri, 2006) if $A \subseteq c l(\operatorname{int}(A))$ and it is called a semi-closed set if $\operatorname{int}(\operatorname{cl}(A)) \subseteq A$

2 pre-open set (Levine, 1963) if $A \subseteq \operatorname{int}(\operatorname{cl}(A))$ and it is called a pre-closed set if $c l(\operatorname{int}(A)) \subseteq A$

$3 \alpha$-open set (Liu, 2008b) if $A \subseteq \operatorname{int}(\operatorname{cl}(\operatorname{int}(A)))$ and it is called a $\alpha$-closed set if $\operatorname{cl}(\operatorname{int}(\operatorname{cl}(A))) \subseteq A$

4 semi-pre-open set (Abu-Donia, 2008) ( $\beta$-open; Pawlak, 1982) if $A \subseteq \operatorname{cl}(\operatorname{int}(\operatorname{cl}(A)))$ and it is called a semi-pre-closed set (Abu-Donia, 2008) ( $\beta$-closed; Abu-Donia, 2008) if $\operatorname{int}(c l(\operatorname{int}(A))) \subseteq A$

5 regular-open set if $A \subseteq \operatorname{int}(c l(A))$ and it is called a regular-closed set if $c l(\operatorname{int}(A)) \subseteq A$

6 semi-regular set (Bryniaski, 1998) if it both semi-open and semi-closed in $(U, \tau)$

$7 \delta$-closed set (Liu and Sai, 2009) if $A \subseteq \operatorname{cl}_{\delta}(A)$, where $c l_{\delta}(A)=\{x \in U$ : $\operatorname{int}(\operatorname{cl}(G)) \cap$ $A \neq \phi, x \in G, G \in \tau\}$.

The semi-closure (resp. $\alpha$-closure, semi-pre-closure) of a subset $A$ of $(U, \tau)$ is the intersection of all semi-closed (resp. $\alpha$-closed, semi-pre-closed) sets that contains $A$ and 
is denoted by $\operatorname{scl}(A)($ resp. $\alpha-\operatorname{cl}(A), \operatorname{spcl}(A))$. The union of all semi-open subsets of $U$ is called the semi-interior of $A$ and is denoted by $\sin t(A)$.

A subset $A$ of a topological space $(U, \tau)$ is called:

1 Generalised closed (briefly $g$-closed) set if $c l(A) \subseteq G$ whenever $A \subseteq G$ and $G \in \tau$.

2 Semi-generalised closed set (briefly $\operatorname{sg}$-closed) if $\operatorname{scl}(A) \subseteq G$ whenever $A \subseteq G$ and $G$ is semi-open set in $(U, \tau)$. The complement of a sg-closed set is called a sg-open set.

3 Generalised semi-closed set (briefly $g s$-closed) if $\operatorname{scl}(A) \subseteq U$ whenever $A \subseteq G$ and $G \in \tau$.

$4 \alpha$-generalised closed set (briefly $\alpha$-closed) if $\alpha c l(A) \subseteq G$ whenever $A \subseteq G$ and $G \in \tau$.

5 Generalised $\alpha$-closed set (briefly g $\alpha$-closed) if $\alpha c l(A) \subseteq G$ whenever $A \subseteq G$ and $G$ is $\alpha$-open in $(U, \tau)$.

$6 g \alpha^{* *}$-closed set if $c l(A) \subseteq \operatorname{int}(\operatorname{cl}(G))$ whenever $A \subseteq G$ and $G$ is $\alpha$-open in $(U, \tau)$.

7 Generalised semi-pre-closed (briefly gsp-closed) set if $\operatorname{spcl}(A) \subseteq G$ whenever $A \subseteq G$ and $G \in \tau$.

$8 \delta$-generalised closed (briefly $\delta g$-closed) set if $c l \delta(A) \subseteq U$ whenever $A \subseteq G$ and $G \in \tau$.

$9 \quad Q$-set if $\operatorname{int}(\operatorname{cl}(A))=\operatorname{cl}(\operatorname{int}(A))$.

\section{Fundamentals of rough sets}

This section presents a review of some fundamental notions of rough sets. We refer to Abu-Donia et al. (2007), Bonikowski (1994), Chuchro (1993), Cattaneo (1998), Deng et al. (2007), Dubois and Prade (1990), Chakrabarty et al. (2000) and Chen and Li (2007) for details.

Motivation for rough set theory has come from the need to represent subsets of a universe in terms of equivalence classes of a partition of that universe. The partition characterises a topological space, called approximation space $A=(U, R)$, where $U$ is a set called the universe and $R$ is an equivalence relation (Biswas, 1994; Chen and Li, 2007). The equivalence classes of $R$ are also known as the granules, elementary sets or blocks; we will use $[x]_{R} \subseteq U$ to denote the equivalence class containing $x \in U$. In the approximation space, we consider two operators $\underline{R}(X)=\left\{x \in U:[x]_{R} \subseteq X\right\}$ and $\bar{R}(X)=$ $\left\{x \in U:[x]_{R} \cap X \neq \phi\right\}$, called the lower approximation and upper approximation of $X \subseteq U$ respectively. Also let $\operatorname{POSR}(X)=\underline{R}(X)$ denote the positive region of $X$, $N E G_{R}(X)=U-\bar{R}(X)$ denote the negative region of $X$ and $B N_{R}(X)=\bar{R}(X)-\underline{R}(X)$ denote the borderline region of $X$.

The degree of completeness can also be characterised by the accuracy measure, in which $X$ represents the cardinality of set $X$ as follows:

$$
\alpha_{R}(X)=|\underline{R}(X)| /|\bar{R}(X)|, \bar{R}(X) \neq \phi .
$$


Accuracy measures try to express the degree of completeness of knowledge. They are able to capture how large the boundary region of the datasets is; however, we cannot easily capture the structure of the knowledge. A fundamental advantage of rough set theory is the ability to handle a category that cannot be sharply defined given a knowledge base. Characteristics of the potential datasets can be measured through the rough sets framework. We can measure inexactness and express topological characterisation of imprecision with:

1 if $\underline{R}(X) \neq \phi$ and $\bar{R}(X) \neq U$, then $X$ is roughly $R$-definable

2 if $\underline{R}(X)=\phi$ and $\bar{R}(X) \neq U$, then $X$ is internally $R$-undefinable

3 if $\underline{R}(X) \neq \phi$ and $\bar{R}(X)=U$, then $X$ is externally $R$-undefinable

4 if $\underline{R}(X)=\phi$ and $\bar{R}(X)=U$, then $X$ is totally $R$-undefinable.

We denote the set of all roughly $R$-definable (resp. internally $R$-undefinable, externally $R$-undefinable and totally $R$-undefinable) sets by $R D(U)$ [resp. $R E U D(U), R U D(U)$ and $R T U D(U)]$.

With $\alpha R(X)$ and classifications above we can characterise rough sets by the size of the boundary region and structure. Rough sets are treated as a special case of relative sets and integrated with the notion of Belmar's logic (Bonikowski, 1994).

\section{Topological approximation spaces and topological rough classifications}

The approximation space $K=(U, R)$ with an equivalence relation $R$ defines a uniquely topological space $\left(U, \tau_{R}\right)$ where $\tau_{R}$ is the quasi-discrete topology with base $U / R$. Moreover, the lower (resp. upper) approximation of any subset $A \subseteq U$ is exactly the interior (resp. closure) of the subset $A \subseteq U$. In this section, we shall generalise Pawlak's concepts in the case of general relations. Also, we will define a new rough approximations using all above topological notions. Hence, the approximation space $K=(U, R)$ with the general relation $R$ defines a uniquely topological space $\left(U, \tau_{R}\right)$.

Let $K=(U, R)$ be an approximation space with general relation $R$ and $\tau_{R}$ is the topology associated with $K=(U, R)$. Then the triple $K_{\tau}=\left(U, R, \tau_{R}\right)$ is called a topological approximation space.

Let $K_{\tau}=\left(U, R, \tau_{R}\right)$ be a topological approximation space. If $X \subseteq U$, then the topological lower approximations of $X$ are defined as follows:

1 semi-lower approximation of $X, \underline{R}_{\text {semi }}(X)=\cup\{G: G \in \operatorname{SEMI}(U), G \subseteq X\}$ where $\operatorname{SEMI}(U)$ is the set of all semi-open sets in $K_{\tau}=\left(U, R, \tau_{R}\right)$

2 pre-lower approximation of $X, \underline{R}_{\text {pre }}(X)=\cup\{G: G \in P R E(U), G \subseteq X\}$ where $\operatorname{PRE}(U)$ is the set of all pre-open sets in $K_{\tau}=\left(U, R, \tau_{R}\right)$

$3 \alpha$-lower approximation of $X, \underline{R}_{\alpha}(X)=\cup\{G: G \in \beta(U), G \subseteq X\}$ where $\alpha(U)$ is the set of all $\alpha$-open sets in $K_{\tau}=\left(U, R, \tau_{R}\right)$

$4 \beta$-lower approximation of $X, \underline{R}_{\beta}(X)=\cup\{G: G \in \beta(U), G \subseteq X\}$ where $\beta(U)$ is the set of all $\beta$-open sets in $K_{\tau}=\left(U, R, \tau_{R}\right)$ 
5 regular-lower approximation of $X, \underline{R}_{\text {regular }}(X)=\cup\{G: G \in R E G(U), G \subseteq X\}$ where $R E G(U)$ is the set of all regular open sets in $K_{\tau}=\left(U, R, \tau_{R}\right)$

6 semi-regular lower approximation of $X, \underline{R}_{\text {semi-regular }}(X)=\cup\{G: G \in \operatorname{SEMI}(U)$ or $G \in \operatorname{CSEMI}(U), G \subseteq X\}$ where $\operatorname{SEMI}(U)$ and $\operatorname{CSEMI}(U)$ are the set of all semi-closed sets in $K_{\tau}=\left(U, R, \tau_{R}\right)$ respectively

$7 \delta$-lower approximation of $X, \underline{R}_{\delta}(X)=\cup\{G: G \in \delta(U), G \subseteq X\}$ where $\delta(U)$ is the set of all $\delta$-closed sets in $K_{\tau}=\left(U, R, \tau_{R}\right)$

$8 g$-lower approximation of $X, \underline{R}_{g}(X)=\cup\{G: G \in g(U), G \subseteq X\}$ where $g(U)$ is the set of all $g$-closed sets in $K_{\tau}=\left(U, R, \tau_{R}\right)$

$9 s g$-lower approximation of $X, \underline{R}_{s g}(X)=\cup\{G: G \in \operatorname{sg}(U), G \subseteq X\}$ where $\operatorname{sg}(U)$ is the set of all $s g$-open sets in $K_{\tau}=\left(U, R, \tau_{R}\right)$

$10 g s$-lower approximation of $X, \underline{R}_{g s}(X)=\cup\{G: G \in g s(U), G \subseteq X\}$ where $g s(U)$ is the set of all $g s$-open sets in $K_{\tau}=\left(U, R, \tau_{R}\right)$

$11 \alpha g$-lower approximation of $X, \underline{R}_{\alpha g}(X)=\cup\{G: G \in \alpha g(U), G \subseteq X\}$ where $\alpha g(U)$ is the set of all $\alpha g$-closed sets in $K_{\tau}=\left(U, R, \tau_{R}\right)$

$12 g \alpha$-lower approximation of $X, \underline{R}_{g \alpha}(X)=\cup\{G: G \in g \alpha(U), G \subseteq X\}$ where $g \alpha(U)$ is the set of all $g \alpha$-closed sets in $K_{\tau}=\left(U, R, \tau_{R}\right)$

$13 g \alpha^{* *}$-lower approximation of $X, \underline{R}_{g \alpha^{* *}}(X)=\cup\left\{G: G \in g \alpha^{* *}(U), G \subseteq X\right.$ where $g \alpha^{* *}(U)$ is the set of all $g \alpha^{* *}$-closed sets in $K_{\tau}=\left(U, R, \tau_{R}\right)$

14 gsp-lower approximation of $X, \underline{R}_{g s p}(X)=\cup\{G: G \in g s p(U), G \subseteq X\}$ where $g s p(U)$ is the set of all gsp-closed sets in $K_{\tau}=\left(U, R, \tau_{R}\right)$

$15 \delta g$-lower approximation of $X, \underline{R}_{\delta g}(X)=\cup\{G: G \in \delta g(U), G \subseteq X\}$ where $\delta g(U)$ is the set of all $\delta g$-closed sets in $K_{\tau}=\left(U, R, \tau_{R}\right)$

$16 Q$-lower approximation of $X, \underline{R}_{Q}(X)=\cup\{G: G \in Q(U), G \subseteq X\}$ where $Q(U)$ is the set of all $Q$-sets in $K_{\tau}=\left(U, R, \tau_{R}\right)$.

All the above topological lower approximations coincide with Pawlak's lower approximation when $R$ is an equivalence relation.

Let $K_{\tau}=\left(U, R, \tau_{R}\right)$ be a topological approximation space. If $X \subseteq U$, then the topological upper approximations of $X$ are defined as follows:

1 semi-upper approximation of $X, \bar{R}_{\text {semi }}(X)=\cup\{F: F \in C \operatorname{SEMI}(U), F \cap X \neq \varphi\}$ where $\operatorname{CSEMI}(U)$ is the set of all semi-closed sets in $K_{\tau}=\left(U, R, \tau_{R}\right)$

2 pre-upper approximation of $X, \bar{R}_{\text {pre }}(X)=\cup\{F: F \in C P R E(U), F \cap X \neq \varphi\}$ where $\operatorname{CPRE}(U)$ is the set of all pre-closed sets in $K_{\tau}=\left(U, R, \tau_{R}\right)$

$3 \alpha$-upper approximation of $X, \bar{R}_{\alpha}(X)=\cup\{F: F \in C \alpha(U), F \cap X \neq \varphi\}$ where $C \alpha(U)$ is the set of all $\alpha$-closed sets in $K_{\tau}=\left(U, R, \tau_{R}\right)$ 
$4 \quad \beta$-upper approximation of $X, \bar{R}_{\beta}(X)=\cup\{F: F \in C \beta(U), F \cap X \neq \varphi\}$ where $C \beta(U)$ is the set of all $\beta$-closed sets in $K_{\tau}=\left(U, R, \tau_{R}\right)$

5 regular-upper approximation of $X, \bar{R}_{\text {regular }}(X)=\cup\{F: F \in C R E G(U), F \cap X \neq \varphi\}$ where $C R E G(U)$ is the set of all regular closed sets in $K_{\tau}=\left(U, R, \tau_{R}\right)$

6 semi-regular upper approximation of $X, \bar{R}_{\text {semi-regular }}(X)=\cup\{F: F \in \operatorname{CSEMI}(U)$, $F \cap X \neq \varphi\}$ where $\operatorname{CSEMI}(U)$ is the set of all semi-closed sets in $K_{\tau}=\left(U, R, \tau_{R}\right)$

$7 \delta$-upper approximation of $X, \bar{R}_{\delta}(X)=\cup\{F: F \in \delta(U), F \cap X \neq \varphi\}$ where $\delta(U)$ is the set of all $\delta$-closed sets in $K_{\tau}=\left(U, R, \tau_{R}\right)$

$8 G$-upper approximation of $X, \bar{R}_{g}(X)=\cup\{F: F \in g(U), F \cap X \neq \varphi\}$ where $g(U)$ is the set of all $g$-closed sets in $K_{\tau}=\left(U, R, \tau_{R}\right)$

9 sg-upper approximation of $X, \bar{R}_{s g}(X)=\cup\{F: F \in C s g(U), F \cap X \neq \varphi\}$ where $\operatorname{Csg}(U)$ is the set of all $\operatorname{sg}$-closed sets in $K_{\tau}=\left(U, R, \tau_{R}\right)$

10 gs-upper approximation of $X, \bar{R}_{g s}(X)=\cup\{F: F \in C g s(U), F \cap X \neq \varphi\}$ where $C g s(U)$ is the set of all gs-closed sets in $K_{\tau}=\left(U, R, \tau_{R}\right)$

$11 \alpha$-upper approximation of $X, \bar{R}_{\alpha g}(X)=\cup\{F: F \in \alpha g(U), F \cap X \neq \varphi\}$ where $\alpha g(U)$ is the set of all $\alpha g$-closed sets in $K_{\tau}=\left(U, R, \tau_{R}\right)$

$12 g \alpha$-upper approximation of $X, \bar{R}_{g \alpha}(X)=\cup\{F: F \in \alpha g(U), F \cap X \neq \varphi\}$ where $g \alpha(U)$ is the set of all $g \alpha$-closed sets in $K_{\tau}=\left(U, R, \tau_{R}\right)$

$13 g \alpha^{* *}$-upper approximation of $X, \bar{R}_{g \alpha^{* *}}(X)=\cup\left\{F: F \in g \alpha^{* *}(U), F \cap X \neq \varphi\right\}$ where $g \alpha^{* *}(U)$ is the set of all $g \alpha^{* *}$-closed sets in $K_{\tau}=\left(U, R, \tau_{R}\right)$

14 gsp-upper approximation of $X, \bar{R}_{g s p}(X)=\cup\{F: F \in g s p(U), F \cap X \neq \varphi\}$ where $g s p(U)$ is the set of all $g s p$-closed sets in $K_{\tau}=\left(U, R, \tau_{R}\right)$

$15 \delta g$-upper approximation of $X, \bar{R}_{\delta g}(X)=\cup\{F: F \in \delta g(U), F \cap X \neq \varphi\}$ where $\delta g(U)$ is the set of all $\delta g$-closed sets in $K_{\tau}=\left(U, R, \tau_{R}\right)$

$16 Q$-upper approximation of $X, \bar{R}_{Q}(X)=\cup\{F: F \in Q(U), F \cap X \neq \varphi\}$ where $Q(U)$ is the set of all $Q$-sets in $K_{\tau}=\left(U, R, \tau_{R}\right)$.

All the above topological upper approximations coincide with Pawlak's upper approximation when $R$ is an equivalence relation.

Motivation for topological rough set theory has come from the need to represent subsets of a universe in terms of topological classes of the topological base generated by the general binary relation defined on the universe. That base characterises a topological space, called topological approximation space $K_{\tau}=\left(U, R, \tau_{R}\right)$. The topological classes of $R$ are also known as the topological granules, topological elementary sets or topological blocks; we will use $G_{x m} \in \tau, m \in\{$ semi, pre, $\alpha, \beta$, regular, semi-regular, $\delta, g, s g$, $\left.\alpha g, g \alpha, g \alpha^{* *}, g s p, \delta g, Q\right\}$ to denote the topological class containing $x \in U$. In the topological approximation space, we consider two operators $\underline{R}_{m}(X)=\left\{x \in U: G_{x m} \subseteq X\right\}$ 
and $\bar{R}_{m}(X)=\left\{x \in U: G_{x m} \cap X \neq \phi\right\}$ called the topological lower approximation and topological upper approximation of $X \subseteq U$ respectively. Also let $\operatorname{POS}_{m}(X)=\underline{R}_{m}(X)$ denotes the topological positive denotes region of $X, N E G_{m}(X)=U-\bar{R}_{m}(X)$ denote the topological negative region of $X$ and $B N D_{m}(X)=\bar{R}_{m}(X)-\underline{R}_{m}(X)$ denote the topological borderline region of $X$.

The degree of topological completeness can also be characterised by the topological accuracy measure, in which $|X|$ represents the cardinality of set $X$ as follows:

$$
\alpha_{m}(X)=\frac{\left|\underline{R}_{m}(X)\right|}{\left|\bar{R}_{m}(X)\right|},
$$

where $X \neq \varphi$.

Topological accuracy measures try to express the degree of completeness of knowledge.

Example 4.1: Let $U=\{a, b, c, d\}$ be a universe and a relation $R$ defined by $R=\{(a, a)$, $(a, c),(a, d),(b, b),(b, d),(c, a),(c, b),(c, d),(d, a)\}$, thus $a R=\{a, c, d\}, b R=\{b, d\}$, $c R=\{a, b, d\}$ and $d R=\{a\}$. Then the topology associated with this relation $R$ is $\tau_{R}=\{U, \varphi,\{a\},\{d\},\{a, d\},\{b, d\},\{a, b, d\},\{a, c, d\}\}$ and $\beta(U)=\{U, \varphi,\{a\},\{d\}$, $\{a, c\},\{a, d\},\{b, d\},\{c, d\},\{a, b, d\},\{a, c, d\},\{b, c, d\}\}$. So $\left(U, R, \tau_{R}\right)$ is a topological approximation space using $\beta$-open sets.

Let $\left(U, R, \tau_{R}\right)$ be a topological approximation space. The universe $U$ can be divided into many regions with respect to any $X \subseteq U$ and with respect to any $m \in$ ssemi, pre, $\alpha$, $\beta$, regular, semi-regular, $\left.\delta, g, s g, \alpha g, g \alpha, g \alpha^{* *}, g s p, \delta g, Q\right\}$ as follows:

1 internal edge of $X$, in-Edge $(X)=X-\bar{R}(X)$

$2 m$-internal edge of $X, m$ - $\underline{\operatorname{Edge}}(X)=X-\underline{R}_{m}(X)$

3 external edge of $X$, ex- $\overline{E d g e}(X)=\bar{R}_{m}(X)-X$

$4 \quad m$-external edge of $X, m$ - $\overline{E d g e}(X)=\bar{R}_{m}(X)-X$

5 boundary of $X, B N(X)=\bar{R}(X)-\underline{R}(X)$

$6 \quad m$-boundary of $X, B N_{m}(X)=\bar{R}_{m}(X)-\underline{R}_{m}(X)$

7 exterior of $X, E X(X)=X-\bar{R}(X)$

$8 m$-exterior of $X, m-E X(X)=X-\bar{R}_{m}(X)$

9 the region $\bar{R}(X)-\underline{R}_{m}(X)$

10 the region $\bar{R}_{m}(X)-\underline{R}(X)$

11 the region $\underline{R}_{m}(X)-\underline{R}(X)$

12 the region $\bar{R}(X)-\bar{R}_{m}(X)$. 
Figure $1 m$-regions of the universe $U$ (see online version for colours)

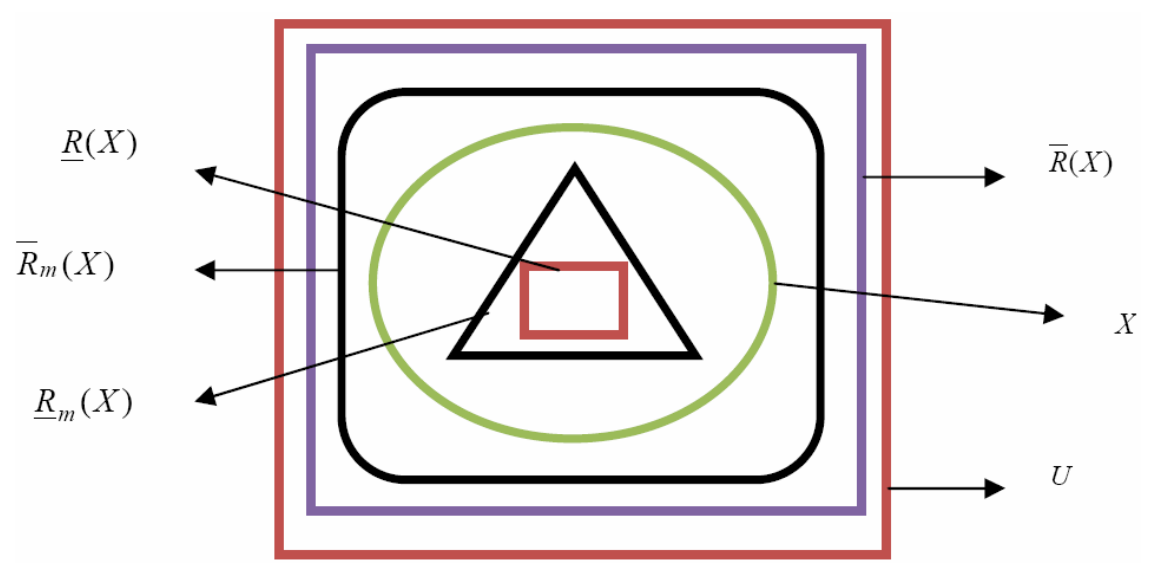

As shown in Figure 1, the study of topological approximation spaces is a generalisation for the study of approximation spaces. Because of the elements of the regions $\underline{R}_{m}(X)-\underline{R}_{m}(X)$ will be defined well in $X$, while this region was indefinable in Pawlak's approximation spaces. Also, the elements of the region $\bar{R}_{m}(X)-\bar{R}_{m}(X)$ do not be belong to $X$, while these elements was not well defined in Pawlak's approximation spaces.

Our aim is to reduce the boundary region of $X$ in Pawlak's approximation space by using $m$-boundary region of $X$. Also, we aim to extend the exterior region of $X$ which contains the elements did not belong to $X$ by $m$-exterior region of $X$.

Proposition 4.1: For any topological approximation space $\left(U, R, \tau_{R}\right)$, the following hold of any $X \subseteq U$ :

$1 B N(X)=\operatorname{in}-\underline{E d g e}(X) \cup \operatorname{ex} \overline{E d g e}(X)$

$2 \quad B N_{m}(X)=m-\underline{E d g e}(X) \cup m-\overline{E d g e}(X)$

$3 \quad \bar{R}(X)-\bar{R}_{m}(X)=e x-\overline{E d g e}(X) \cup m-\underline{E d g e}(X)$

$4 \quad \bar{R}_{m}(X)-\bar{R}(X)=m-\overline{E d g e}(X) \cup \operatorname{in}-\underline{E d g e}(X)$

$5 \quad \operatorname{in}-\operatorname{Edge}(X)=m-\operatorname{Edge}(X) \cup\left(\underline{R}_{m}(X)-\underline{R}(X) \underline{R}_{m}(X)-\underline{R}(X)\right)$

$6 \quad e x-\overline{E d g e}(X)=m-\underline{E d g e}(X) \cup\left(\bar{R}(X)-\bar{R}_{m}(X)\right)$.

Proof: See Figure 1.

Let $\left(U, R, \tau_{R}\right)$ be a topological approximation space. For any $m \in\{$ semi, pre, $\alpha, \beta$, regular, semi-regular, $\left.\delta, g, s g, \alpha g, g \alpha, g \alpha^{* *}, g s p, \delta g, Q\right\}$ and for any subset $X \subseteq U$ we define the following memberships:

$1 x$ belongs strong to $X$ if $x \in \underline{R}(X)$

$2 x$ belongs weak to $X$ if $x \in \bar{R}(X)$ 
$3 x$ belongs $m$-strong to $X$ if $x \in \underline{R}_{m}(X)$

$4 x$ belongs $m$-weak to $X$ if $x \in \bar{R}_{m}(X)$.

Example 4.2: Let $U=\{a, b, c, d\}$ be a universe and a relation $R$ defined by $R=\{(a, a)$, $(d, c),(d, d),(c, a),(c, d),(c, c)\}$, thus $a R=\{a\}, b R=\varphi, c R=\{a, c, d\}$ and $d R=\{c, d\}$. Then the topology associated with this relation is $\tau_{R}=\{U, \varphi,\{a\},\{c, d\},\{a, c, d\}\}$. So $\left(U, R, \tau_{R}\right)$ is a topological approximation space. Let $X=\{b, c, d\}$, we have $b$ is $m$-strong belongs to $X$ but $b$ is not strong belongs to $X$. Also, let $Y=\{c\}$ be another subset of $U$. Then we have $d$ is weak belongs to $Y$ but $d$ is not $m$-weak belongs to $Y$.

The degree of topological completeness can also be characterised by the topological accuracy measure ( $m$-accuracy), in which $|X|$ represents the cardinality of set $X$ as follows: $\alpha_{m}(X)=\left|\underline{R}_{m}(X)\right| /\left|\bar{R}_{m}(X)\right|$, where $X \neq \varphi$.

According to Example 4.1, Table 1 showing the differences among the degree of Pawlak's accuracy measure $\alpha(X)$ and $\beta$-accuracy measure $\alpha_{\beta}(X)$ for some subsets of $U$ if we take $m=\beta$.

Table 1 Comparison between Pawlak's accuracy measure and $\beta$-accuracy measure

\begin{tabular}{lcc}
\hline$X$ & $\alpha(X)$ (Pawlak) & $\alpha_{\beta}(X)$ \\
\hline$\{a\}$ & $50 \%$ & $100 \%$ \\
$\{a, c\}$ & $50 \%$ & $100 \%$ \\
$\{b, d\}$ & $33.3 \%$ & $100 \%$ \\
$\{b, c, d\}$ & $66.6 \%$ & $100 \%$ \\
\hline
\end{tabular}

The $\beta$-accuracy measure of the class of all $\beta$-open sets is accurate than the other measures and the following example and its followed diagram illustrate this fact.

Example 4.3: Let $U=\{a, b, c, d\}$ be a universe and for some relations we have the topology $\tau_{R}=\{U, \varphi,\{d\},\{a, b\},\{a, b, d\}\}$. So $\left(U, R, \tau_{R}\right)$ is a topological approximation space. Then we have the following knowledge bases:

$$
\begin{aligned}
& \operatorname{REG}(U)=\{U, \varphi,\{d\},\{a, b\}\}, \\
& \operatorname{SEMI}(U)=\{U, \varphi,\{d\},\{a, b\},\{c, d\},\{a, b, c\},\{a, b, d\}\}, \\
& \operatorname{PRE}(U)=\{U, \varphi,\{a\},\{b\},\{d\},\{a, d\},\{a, b\},\{b, d\},\{a, c, d\},\{a, b, d\},\{b, c, d\}\}, \\
& \alpha(U)=\{U, \varphi,\{d\},\{a, b\},\{a, b, d\}\}, \\
& \beta(U)=\left\{\begin{array}{l}
U, \varphi,\{a\},\{b\},\{d\},\{a, d\},\{a, b\},\{a, c\},\{b, d\}, \\
\{b, c\},\{c, d\},\{a, b, c\},\{a, c, d\},\{a, b, d\},\{b, c, d\}
\end{array}\right\} .
\end{aligned}
$$

Figure 2 illustrated the relationships among these knowledge bases. 
Figure 2 Some knowledge bases of the universe $U$ (see online version for colours)

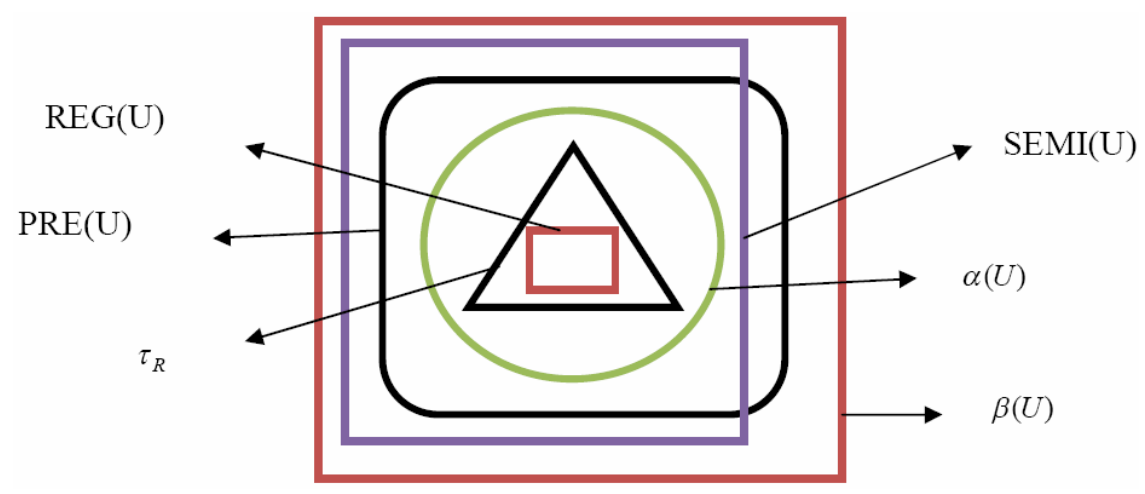

Proposition 4.2: For any topological approximation space $K_{\tau}=\left(U, R, \tau_{R}\right)$, the following are hold for any $X, Y \subseteq U$ :

$1 \quad \underline{R}_{m}(X \cup Y) \supseteq \underline{R}_{m}(X) \cup \underline{R}_{m}(Y)$

$2 \quad \bar{R}_{m}(X \cup Y) \supseteq \bar{R}_{m}(X) \cup \underline{R}_{m}(Y)$

$3 \quad \underline{R}_{m}(X \cap Y) \subseteq \underline{R}_{m}(X) \cap \underline{R}_{m}(Y)$

$4 \quad \bar{R}_{m}(X \cap Y) \subseteq \bar{R}_{m}(X) \cap \underline{R}_{m}(Y)$.

Proof: We prove Part 1 and other parts are similar to it.

Since we have $X \subseteq X \cup Y$ and $Y \subseteq X \cup Y$. Then $\underline{R}_{m}(X) \subseteq \underline{R}_{m}(X \cup Y)$ and $\underline{R}_{m}(Y) \subseteq \underline{R}_{m}(X \cup Y)$ then $\underline{R}_{m}(X \cup Y) \supseteq \underline{R}_{m}(X) \cup \underline{R}_{m}(Y)$. The equality of all parts in the above proposition is not holding as shown in the following example.

Example 4.3: According to Example 4.1 we have:

- If $X=\{d\}, Y=\{a, b\}$, then we have $\underline{R}_{m}(X \cup Y)=\{a, b, d\}, \underline{R}_{m}(X)=\{d\}$, $\underline{R}_{m}(Y)=\{a\}$. Therefore, $\underline{R}_{m}(X \cup Y) \neq \underline{R}_{m}(X) \cup \bar{R}_{m}(Y)$.

- If $X=\{d\}, Y=\{a, b\}$, then we have $\bar{R}_{m}(X \cup Y)=\{a, b, c, d\}, \bar{R}_{m}(X)=\{b, d\}$, $\bar{R}_{m}(Y)=\{a, b\}$. Therefore, $\bar{R}_{m}(X \cup Y) \neq \bar{R}_{m}(X) \cup \bar{R}_{m}(Y)$.

- If $X=\{a, b, c\}, Y=\{b, c, d\}$, then we have $\underline{R}_{m}(X \cap Y)=\varphi, \underline{R}_{m}(X)=\{a, c\}$ $\underline{R}_{m}(Y)=\{b, c, d\}$. Therefore, $\underline{R}_{m}(X \cap Y) \neq \underline{R}_{m}(X) \cap \underline{R}_{m}(Y)$.

- If $X=\{b\}, Y=\{c, d\}$, then we have $\bar{R}_{m}(X \cap Y)=\varphi, \bar{R}_{m}(X)=\{b\}, \bar{R}_{m}(Y)=\{b, c, d\}$. Therefore, $\bar{R}_{m}(X \cap Y) \neq \bar{R}_{m}(X) \cap \bar{R}_{m}(Y)$. 


\section{Many m-topological approximations}

We will give the deviation to four concepts namely, membership, equality, inclusion and power set of our approach using any $m$-open set.

Definition 5.1: Let $K_{\tau}=\left(U, R, \tau_{R}\right)$ be a topological approximation space and $X \subseteq U$. Then we say that:

$1 x$ is $\tau_{R}$-surely belongs to $X$, written $x \underline{\epsilon}_{\tau} X$, if $x \in \underline{R}_{m}(X)$

$2 x$ is $\tau_{R}$-possibly belongs to $X$, written $x \bar{\epsilon}_{\tau} X$, if $x \in \bar{R}_{m}(X)$.

These two membership relations $\in_{\tau}$ and $\bar{\epsilon}_{\tau}$ are called $\tau_{R^{2}}$-strong and $\tau_{R^{-}}$-weak membership relations respectively and it is clear that $x \underline{\epsilon}_{\tau} X$ implies to $x \in X$ and $x \in X$ implies to $x \bar{\epsilon}_{\tau} X$.

The converse is not true as we illustrate with the following example:

Example 5.1: Let $U=\{a, b, c, d\}$ be the universe of discourse and $R$ is a binary relation on $U$ such that: $a R=\{a\}, b R=\{b\}, c R=\{b, c, d\}$ and $d R=\{a\}$, then $\tau_{R}=\{U, \varphi,\{a\}$, $\{b\},\{a, b\},\{a, d\},\{a, b, d\}\}$, let $X=\{a, c\}$, then $\underline{R}_{m}(X)=\{a\}$ and $\bar{R}_{m}(X)=\{a, c, d\}$ it is clear that $c \in X$ but $c \notin \underline{R}_{m}(X)$, i.e., non- $c \in_{\tau} X$ and also $d \in \bar{R}_{m}(X)$, i.e., $d \bar{\epsilon}_{\tau} X$ but $d \notin X$.

Proposition 5.1: Let $K_{\tau}=\left(U, R, \tau_{R}\right)$ be a topological approximation space and $X, Y \subseteq U$. Then by using the properties of approximations we can prove the following facts:

1 if $X \subseteq Y$, then ( $x \underline{\epsilon}_{\tau} X$ implies to $x \underline{\epsilon}_{\tau} Y$ and $x \bar{\epsilon}_{\tau} X$ implies to $x \bar{\epsilon}_{\tau} Y$ )

$2 x \bar{\epsilon}_{\tau}(X \cup Y)$ if and only if $x \bar{\epsilon}_{\tau} X$ or $x \bar{\epsilon}_{\tau} Y$

$3 x \bar{\epsilon}_{\tau}(X \cap Y)$ if and only if $x \bar{\epsilon}_{\tau} X$ and $x \bar{\epsilon}_{\tau} Y$

4 if $x \underline{\in}_{\tau} X$ or $x \underline{\in}_{\tau} Y$, then $x \underline{\in}_{\tau}(X \cup Y)$

5 if $x \underline{\epsilon}_{\tau}(X \cap Y)$ then $x \underline{\epsilon}_{\tau} X$ and $x \underline{\epsilon}_{\tau} Y$

$6 x \underline{\epsilon}_{\tau}(-X)$ if and only if non- $x \bar{\epsilon}_{\tau} X$

$7 x \bar{\epsilon}_{\tau}(-X)$ if and only if non- $x \underline{\epsilon}_{\tau} X$.

Remark 5.1: In the case of $R$ is an equality relation, all these memberships relations $\underline{\epsilon}_{\tau}$ and $\bar{\epsilon}_{\tau}$ are the same and coincides with ordinary membership relation $\in$. We can redefine the topological rough approximations by using $\underline{\epsilon}_{\tau}$ and $\bar{\epsilon}_{\tau}$ as follow: For any $X \subseteq U, \underline{R}(X)=\left\{x \in U: x \underline{\in}_{\tau} X\right\}$ and $\bar{R}(X)=\left\{x \in U: x \bar{\epsilon}_{\tau} X\right\}$.

Definition 5.2: Let $K_{\tau}=\left(U, R, \tau_{R}\right)$ be a topological approximation space. Then the two subset $X, Y \subseteq U$ are called:

$1 \quad \tau_{R}$-roughly bottom-equal in $K_{\tau}=\left(U, R, \tau_{R}\right)$, written $X \bar{\sim}_{\tau} Y$, if $\underline{R}_{m}(X)=\underline{R}_{m}(Y)$

$2 \tau_{R}$-roughly top-equal in $K_{\tau}=\left(U, R, \tau_{R}\right)$, written $X \simeq_{\tau} Y$, if $\bar{R}_{m}(X)=\bar{R}_{m}(Y)$ 
$3 \tau_{R}$-roughly equal in $K_{\tau}=\left(U, R, \tau_{R}\right)$, written $X \approx_{\tau} Y$, if $X \bar{\sim}_{\tau} Y$ and $X \simeq_{\tau} Y$.

Definition 5.3: Let $K_{\tau}=\left(U, R, \tau_{R}\right)$ be a topological approximation space. Then the subset $X \subseteq U$ is said to be:

$1 \tau_{R}$-dense in $K_{\tau}=\left(U, R, \tau_{R}\right)$ if $X \simeq_{\tau} U$

$2 \tau_{R}$-co-dense in $K_{\tau}=\left(U, R, \tau_{R}\right)$ if $X \bar{\sim}_{\tau} \phi$

$3 \tau_{R^{-}}$dispersed in $K_{\tau}=\left(U, R, \tau_{R}\right)$ if $X \simeq_{\tau} U$ and $X \bar{\sim}_{\tau} \phi$.

Two different sets which are not equal in ordinary set theory, can be equal (approximately) in our generalised topological rough sets as the following example illustrates:

Example 5.2: Consider $U=\{a, b, c, d, e\}$ be the universe of discourse and $R$ is a binary relation on $U$ where $a R=\{a\}, b R=\{c, d\}, c R=\{e, d\}, d R=\{d, d\}$ and $e R=\{e\}$. Then $\tau_{R}=\{U, \phi,\{a\},\{e\},\{a, d\},\{a, e\},\{a, c, e\},\{a, d, e\},\{a, c, d, e\}\}$.

Let $X_{1}=\{a, c, d\}, Y_{1}=\{a, b, d\}, X_{2}=\{b, c, d\}$ and $Y_{2}=\{a, b, d, e\}$. Then $\underline{R}_{m}\left(X_{1}\right)=\{a, b\}=\underline{R}_{m}\left(Y_{1}\right)$, i.e., $X_{1} \bar{\sim}_{\tau} Y_{1}$ and $\bar{R}_{m}\left(X_{1}\right)=\{a, b, c, d\}=\bar{R}_{m}\left(Y_{1}\right)$, i.e., $X_{1} \simeq_{\tau} Y_{1}$. Thus, $X_{1} \approx_{\tau} Y_{1}$ although $X_{1} \neq Y_{1}$. Also $\underline{R}_{m}\left(X_{2}\right)=\phi$ and $\bar{R}_{m}\left(Y_{2}\right)=U$, then $X_{2} \bar{\sim}_{\tau} \phi$ and $Y_{2} \simeq_{\tau} U$ that is $Y_{2}$ is $\tau_{R}$-dense and $X_{2}$ is $\tau_{R}$-co-dense in $K_{\tau}=\left(U, R, \tau_{R}\right)$.

Proposition 5.2: Let $K_{\tau}=\left(U, R, \tau_{R}\right)$ be a topological approximation space and $X, Y, X^{\prime}$, $Y^{\prime} \subseteq U$. Then:

1 if $X \simeq_{\tau} Y$, then $(X \cup Y) \simeq_{\tau} X \simeq_{\tau} Y$

2 if $X \bar{\sim}_{\tau} Y$, then $(X \cap Y) \bar{\sim}_{\tau} X \bar{\sim}_{\tau} Y$

3 if $X \simeq_{\tau} X^{\prime}$ and $Y \simeq_{\tau} Y^{\prime}$, then $(X \cup Y) \simeq_{\tau}\left(X^{\prime} \cup Y^{\prime}\right)$

4 if $X \bar{\sim}_{\tau} X^{\prime}$ and $Y \bar{\sim}_{\tau} Y^{\prime}$, then $(X \cap Y) \simeq_{\tau}\left(X^{\prime} \cap Y^{\prime}\right)$.

Proof:

1 Let $X \simeq_{\tau} Y$, then $\bar{R}_{m}(X)=\bar{R}_{m}(Y)$. But $\bar{R}_{m}(X \cup Y)=\bar{R}_{m}(X) \cup \bar{R}_{m}(Y)$. Then $\bar{R}_{m}(X \cup Y)=\bar{R}_{m}(X) \cup \bar{R}_{m}(X)=\bar{R}_{m}(X)$, and $\bar{R}_{m}(X \cup Y)=\bar{R}_{m}(Y) \cup \bar{R}_{m}(Y)=\bar{R}_{m}(Y)$, hence $(X \cup Y) \simeq_{\tau} X \simeq_{\tau} Y$.

2 Similarly as (1).

3 Let $X \simeq_{\tau} X^{\prime}$ and $Y \simeq_{\tau} Y^{\prime}$, then $\bar{R}_{m}(X)=\bar{R}_{m}\left(X^{\prime}\right)$ and $\bar{R}_{m}(Y)=\bar{R}_{m}\left(Y^{\prime}\right)$. Thus, $\bar{R}_{m}(X) \cup \bar{R}_{m}(Y)=\bar{R}_{m}\left(X^{\prime}\right) \cup \bar{R}_{m}\left(Y^{\prime}\right)$, which implies that $\bar{R}_{m}(X \cup Y)=\bar{R}_{m}\left(X^{\prime} \cup Y^{\prime}\right)$. Thus, $(X \cup Y) \simeq\left(X^{\prime} \cup Y^{\prime}\right)$.

4 By similar way as in (3).

Proposition 5.3: Let $K_{\tau}=\left(U, R, \tau_{R}\right)$ be a topological approximation space and $X, Y \subseteq U$. Then: 
$1 \quad X$ is $\tau_{R^{-}}$-dense if and only if $(-X)$ is $\tau_{R^{-c o}}$-dense

$2 X$ is $\tau_{R}$-dispersed if and only if $(-X)$ is $\tau_{R}$-dispersed

3 any superset of $\tau_{R}$-dense set is also $\tau_{R^{-}}$-dense

4 any subset of $\tau_{R}$-co-dense set is also $\tau_{R}$-co-dense.

Proof:

1 Let $X$ is dense in $K_{\tau}=\left(U, R, \tau_{R}\right)$ if and only if $X \simeq_{\tau} U$ iff $\underline{R}_{m}(X)=\underline{R}_{m}(U)$. But $\underline{R}_{m}(X)=-\underline{R}_{m}(-X)$, hence $X$ is $\tau_{R}$-dense iff $-\underline{R}_{m}(-X)=-\underline{R}_{m}(-U)=-\underline{R}_{m}(\phi)$ iff $\underline{R}_{m}(-X)=\underline{R}_{m}(\phi)$ iff $(-X)$ is $\tau_{R}$-co-dense.

2 Let $X$ is $\tau_{R^{-}}$dispersed in $K_{\tau}=\left(U, R, \tau_{R}\right)$ iff $X$ is $\tau_{R^{-}}$-dense and $\tau_{R^{-}}$-co-dense iff $(-X)$ is $\tau_{R}$-co-dense and $\tau_{R}$-dense iff $(-X)$ is $\tau_{R}$-dispersed

3 Let $Y$ is a superset of $X$ and $X$ is $\tau_{R}$-dense. Then $X \subseteq Y$ and $X \simeq_{\tau} U$, hence $\bar{R}_{m}(X) \subseteq \bar{R}_{m}(Y)$ and $\bar{R}_{m}(X)=\bar{R}_{m}(U)=U$, which means that $U \subseteq \bar{R}_{m}(Y)$, but $\bar{R}_{m}(Y) \subseteq U$ then $\bar{R}_{m}(Y)=U=\bar{R}_{m}(U)$, that is $Y \simeq_{\tau} U$ and $Y$ is $\tau_{R}$-dense.

4 By similar way as in (3).

Proposition 5.4: Let $K_{\tau}=\left(U, R, \tau_{R}\right)$ be a topological approximation space, then the lower (resp. the upper) approximation of any subset $X \subseteq U, \underline{R}_{m}(X)$ (resp. $\bar{R}_{m}(X)$ ) is the

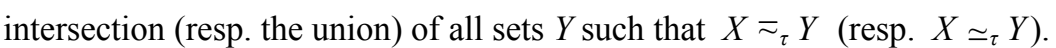

Proof: First, let $Y \subseteq U$ such that $X \bar{\sim}_{\tau} Y$, then $\underline{R}_{m}(X)=\underline{R}_{m}(Y)$. But $\underline{R}_{m}(Y) \subseteq Y$,

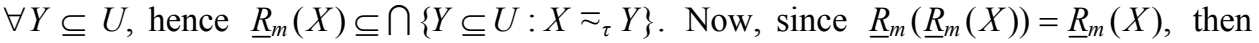

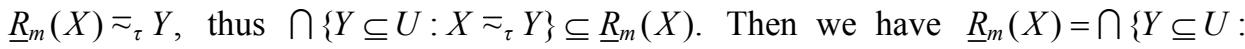
$\left.X \approx_{\tau} Y\right\}$. The last part of the proof is by similar way.

Proposition 5.5: Let $K_{\tau}=\left(U, R, \tau_{R}\right)$ be a topological approximation space and $X, Y \subseteq U$. Then:

$1 \quad X \simeq_{\tau} Y$ if and only if $(-X) \bar{\tau}_{\tau}(-Y)$

2 if $X \bar{\sim}_{\tau} \phi$ or $Y \bar{\sim}_{\tau} \phi$, then $(X \cap Y) \bar{\sim}_{\tau} \phi$

3 if $X \simeq_{\tau} U$ or $Y \simeq_{\tau} U$, then $(X \cup Y) \simeq_{\tau} U$.

Proof:

1 Is obvious.

2 Let $X \bar{\sim}_{\tau} \phi$ or $Y \bar{\sim}_{\tau} \phi$, then $\underline{R}_{m}(X)=\underline{R}_{m}(\phi)=\phi$ or $\underline{R}_{m}(Y)=\underline{R}_{m}(\phi)=\phi$. Thus, $\underline{R}_{m}(X \cap Y)=\underline{R}_{m}(X) \cap \underline{R}_{m}(Y)=\phi=\underline{R}_{m}(\phi)$, that is $(X \cap Y) \bar{\sim}_{\tau} \phi$.

3 By similar way as in 2. 
Proposition 5.6: The rough equalities $\bar{\sim}_{\tau}, \simeq_{\tau}$ and $\approx_{\tau}$ are equivalence relations on the power set $P(U)$ in $K_{\tau}=\left(U, R, \tau_{R}\right)$.

Proof: Clearly, for any $X \subseteq U, \underline{R}_{m}(X)=\underline{R}_{m}(X)$ and $\bar{R}_{m}(X)=\bar{R}_{m}(X)$, thus $X \bar{\sim}_{\tau} X$, $X \simeq_{\tau} X$ and $X \approx_{\tau} X$, and thus $\bar{\sim}_{\tau}, \simeq_{\tau}$ and $\approx_{\tau}$ are reflexive relations. Let $X{\sim_{\tau}}_{\tau}$, $X \simeq_{\tau} Y$ and $X \approx_{\tau} Y$, then $\underline{R}_{m}(X)=\underline{R}_{m}(Y)$ and $\bar{R}_{m}(X)=\bar{R}_{m}(Y)$, then $Y{\approx_{\tau}}_{\tau} X, Y \simeq_{\tau} X$ and $Y \approx_{\tau} X$, that is $\bar{\sim}_{\tau}, \simeq_{\tau}$ and $\approx_{\tau}$ are symmetric relations. If $X{{ }_{\tau}}_{\tau} Y$ and $Y{\sim_{\tau}}_{\tau} Z$, then $\underline{R}_{m}(X)=\underline{R}_{m}(Y)$ and $\bar{R}_{m}(Y)=\bar{R}_{m}(Z)$, and thus $\underline{R}_{m}(X)=\underline{R}_{m}(Z)$ and $X \bar{\sim}_{\tau} Z$, that is $\bar{\sim}_{\tau}, \simeq_{\tau}$ and $\approx_{\tau}$ are transitive relations. Thus, $\bar{\sim}_{\tau}, \simeq_{\tau}$ and $\approx_{\tau}$ are equivalence relations.

Definition 5.4: Let $K_{\tau}=\left(U, R, \tau_{R}\right)$ be a topological approximation space and $X, Y \subseteq U$. We say that:

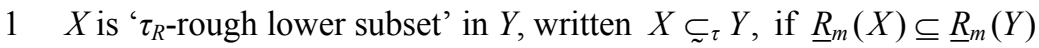

$2 X$ is ' $\tau_{R}$-rough upper subset' in $Y$, written $X \tilde{\subset}_{\tau} Y$, if $\bar{R}_{m}(X) \subseteq \bar{R}_{m}(Y)$

$3 X$ is ' $\tau_{R}$-rough subset' in $Y$, written $X \tilde{\sim}_{\tau} Y$, if $X \bigodot_{\tau} Y$ and $Y \subset_{\tau} X$.

The rough inclusion of sets does not imply the inclusion of sets as the following example illustrates.

Example 5.3: Consider $U=\{a, b, c, d\}$ be the universe of discourse and $R$ is a binary relation to $U$, where $a R=\{a\}, b R=\{b\}, c R=\{b, c\}$ and $d R=\{a\}$. Then $\tau_{R}=\{U, \phi,\{a\}$, $\{b\},\{a, b\},\{a, d\},\{b, c\},\{a, b, c\},\{a, b, d\}\}$ and let $X=\{b, c\}$ and $Y=\{a, b, d\}$, clearly $X \not \subset Y$ and we have $\underline{R}_{m}(X)=\{b\}, \underline{R}_{m}(X)=\{a, b, d\}, \underline{R}_{m}(X)=\{b, c, d\}$ and $\bar{R}_{m}(Y)=U$. Then $X \subset_{\tau} Y$ and $X \tilde{\subset}_{\tau} Y$ which implies that $X \tilde{\sim}_{\tau} Y$ although $X \not \subset Y$.

Proposition 5.7: Let $K_{\tau}=\left(U, R, \tau_{R}\right)$ be a topological approximation space and $X, Y \subseteq U$. Then

1 If $X \subseteq Y$, then $X \sim_{\tau} Y, X \tilde{\subset}_{\tau} Y$ and $X \tilde{\sim}_{\tau} Y$

$2 X \subset_{\tau} Y$ and $Y \subset_{\tau} X$ if and only if $X{{ }_{\tau}}_{\tau} Y$

$3 X \tilde{\subset}_{\tau} Y$ and $Y \tilde{\subset}_{\tau} X$ if and only if $X \simeq_{\tau} Y$

$4 X \tilde{\sim}_{\tau} Y$ and $Y \tilde{\sim}_{\tau} X$ if and only if $X \approx_{\tau} Y$.

Proof:

1 Is obvious.

2 Let $X \subseteq_{\tau} Y$ and $Y \subseteq_{\tau} X$ iff $\underline{R}_{m}(X) \subseteq \underline{R}_{m}(Y)$ and $\underline{R}_{m}(Y) \subseteq \underline{R}_{m}(X)$ iff $\underline{R}_{m}(X)=\underline{R}_{m}(Y)$ iff $X \bar{\sim}_{\tau} Y$.

The proof of Parts 3 and 4 are similar as Part 2.

Proposition 5.8: Let $K_{\tau}=\left(U, R, \tau_{R}\right)$ be a topological approximation space and $X, Y, X^{\prime}, Y^{\prime}$ $\subseteq U$. Then: 
$1 X \subset_{\tau} Y$ if and only if $(X \cap Y) \bar{\sim}_{\tau} X$

$2 X \tilde{\subset}_{\tau} Y$ if and only if $(X \cup Y) \simeq_{\tau} Y$

$3(X \cap Y) \subseteq_{\tau} X \tilde{\subset}_{\tau}(X \cup Y)$

4 if $X \subseteq Y, X \bar{\sim}_{\tau} X^{\prime}$ and $Y \bar{\sim}_{\tau} Y^{\prime}$, then $X^{\prime} \subseteq_{\tau} Y^{\prime}$

5 if $X \subseteq Y, X \simeq_{\tau} X^{\prime}$ and $Y \simeq_{\tau} Y^{\prime}$, then $X^{\prime} \tilde{\complement}_{\tau} Y^{\prime}$

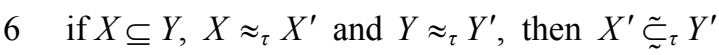

7 if $X^{\prime} \tilde{\subset}_{\tau} X$ and $Y^{\prime} \tilde{\subset}_{\tau} Y$, then $\left(X^{\prime} \cup Y^{\prime}\right) \tilde{\subset}_{\tau}(X \cup Y)$

8 if $X^{\prime} \varsigma_{\tau} X$ and $Y^{\prime} \varsigma_{\tau} Y$, then $\left(X^{\prime} \cap Y^{\prime}\right) \subsetneq_{\tau}(X \cap Y)$.

Proof:

$1 \quad$ Let $X \subset_{\sim} Y$ iff $\underline{R}_{m}(X) \subseteq \underline{R}_{m}(Y)$ iff $\underline{R}_{m}(X) \cap \underline{R}_{m}(Y)=\underline{R}_{m}(X)$ iff $\underline{R}_{m}(X \cap Y)=\underline{R}_{m}(X)$ iff $(X \cap Y) \bar{\tau}_{\tau} X$.

2 By similar way as in 1.

3 Since $\underline{R}_{m}(X \cap Y)=\underline{R}_{m}(X) \cap \underline{R}_{m}(Y)$ and $\underline{R}_{m}(X \cup Y)=\underline{R}_{m}(X) \cup \underline{R}_{m}(Y)$. Then $\underline{R}_{m}(X \cap Y) \subseteq \underline{R}_{m}(X)$ and $\bar{R}_{m}(X) \subseteq(X \cup Y)$, and hence $(X \cap Y) \subseteq_{\tau} X \tilde{\subset}_{\tau}(X \cup Y)$.

4 Let $X \subseteq Y, X{\approx_{\tau}} X^{\prime}$ and $Y \simeq_{\tau} Y^{\prime}$, then $\underline{R}_{m}(X) \subseteq \underline{R}_{m}(Y), \underline{R}_{m}(X)=\underline{R}_{m}\left(X^{\prime}\right)$ and $\underline{R}_{m}(Y)=\underline{R}_{m}\left(Y^{\prime}\right)$. Thus, $\underline{R}_{m}\left(X^{\prime}\right) \subseteq \underline{R}_{m}\left(Y^{\prime}\right)$ and then $X^{\prime} \subseteq_{\sim} Y^{\prime}$.

5 and 6 by similar way as in 4 .

7 Let $X^{\prime} \tilde{\complement}_{\tau} X$ and $Y^{\prime} \tilde{\complement}_{\tau} Y$, then $\bar{R}_{m}\left(X^{\prime}\right) \subseteq \bar{R}_{m}(X)$ and $\bar{R}_{m}\left(Y^{\prime}\right) \subseteq \bar{R}_{m}(X)$, hence $\bar{R}_{m}\left(X^{\prime}\right) \cup \bar{R}_{m}\left(Y^{\prime}\right) \subseteq \bar{R}_{m}(X) \cup \bar{R}_{m}(Y)$, and $\bar{R}_{m}\left(X^{\prime} \cup Y^{\prime}\right) \subseteq \bar{R}_{m}(X \cup Y)$. That is $\left(X^{\prime} \cup Y^{\prime}\right) \tilde{\subset}_{\tau}(X \cup Y)$.

8 By similar way as in (7).

Proposition 5.9: Let $K_{\tau}=\left(U, R, \tau_{R}\right)$ be a topological approximation space and $X, Y, Z \subseteq$ $U$. Then

1 if $X \subset_{\tau} Y$ and $X \bar{\sim}_{\tau} Z$, then $Z \bigodot_{\tau} Y$

2 if $X \tilde{\subset}_{\tau} Y$ and $X \simeq_{\tau} Z$, then $Z \tilde{\subset}_{\tau} Y$

3 if $X \tilde{\sim}_{\tau} Y$ and $X \approx_{\tau} Z$, then $Z \tilde{\sim}_{\tau} Y$.

Proof: Obvious.

Proposition 5.10: The rough inclusion relations $\subseteq_{\tau}, \tilde{\complement}_{\tau}$ and $\tilde{\sim}_{\tau}$ are represented ordering relations on $P(U)$.

Proof: Obvious. 
The following theorems study the properties and relationships among boundary, positive and negative regions in Pawlak's approach and in our topological approximation space.

Theorem 5.1: Let $K_{\tau}=\left(U, R, \tau_{R}\right)$ be a topological approximation space and for any subset $X \subset U$ we have:

$1 \quad B N D_{m}(X) \cap \underline{R}_{m}(X)=\varphi$

$2 B N D_{m}(X) \cap N E G_{m}(X)=\varphi$

$3 \quad B N D_{m}(X) \cup \underline{R}_{m}(X)=\bar{R}_{m}(X)$

$4 \underline{R}_{m}(X), N E G_{m}(X)$ and $B N D_{m}(X)$ form a partition of $U$.

Proof: You can make use of Figure 1.

Theorem 5.2: Let $K_{\tau}=\left(U, R, \tau_{R}\right)$ be a topological approximation space and for any subsets $X, Y \subset U$ we have:

$1 B N D_{m}(U)=\varphi$

$2 B N D_{m}(X)=B N D_{m}(U-X)$

$3 \quad B N D_{m}\left(B N D_{m}(X)\right) \subset B N D_{m}(X)$

$4 \quad B N D_{m}(X \cap Y) \subset B N D_{m}(X) \cup B N D_{m}(Y)$.

Proof: 1 and 2 are obvious, by the definitions.

$3 B N D_{m}\left(B N D_{m}(X)\right)=B N D_{m}\left(\bar{R}_{m}(X) \cap \bar{R}_{m}(U-X)\right)$ $=\bar{R}_{m}\left(\bar{R}_{m}(X) \cap \bar{R}_{m}(U-X)\right.$ $\cap \bar{R}_{m}\left(U-\left(\bar{R}_{m}(X) \cap \bar{R}_{m}(U-X)\right)\right.$ $\subset \bar{R}_{m}(X) \cap \bar{R}_{m}(U-X)$ $=B N D_{m}(X)$.

$4 \quad B N D_{m}(X \cap Y)=\bar{R}_{m}(X \cap Y) \cap \bar{R}_{m}(U-X \cap Y)$.

Theorem 5.3: Let $K_{\tau}=\left(U, R, \tau_{R}\right)$ be a topological approximation space and for any subset $X, Y \subset U$ we have:

$1 \quad U=N E G_{m}(\varphi)$

$2 \quad N E G_{m}(X)=\underline{R}_{m}(U-X)$

$3 X \cap N E G_{m}(X)=\varphi$

$4 \quad N E G_{m}\left(U-N E G_{m}(X)\right)=N E G_{m}(X)$

$5 \quad N E G_{m}(X \cup Y) \subset N E G_{m}(X) \cup N E G_{m}(Y)$

$6 N E G_{m}(X \cap Y) \supset N E G_{m}(X) \cap N E G_{m}(Y)$.

Proof: 1, 2, 3 and 4 are obvious. 
$5 \quad N E G_{m}(X \cup Y)=U-\bar{R}_{m}(X \cup Y) \subset U-\left(\bar{R}_{m}(X) \cup \bar{R}_{m}(Y)\right)$

$$
=\left(U-\bar{R}_{m}(X)\right) \cap\left(U-\bar{R}_{m}(Y)\right) \subset N E G_{m}(X) \cup N E G_{m}(Y) .
$$

$6 N E G_{m}(X) \cap N E G_{m}(Y)=\left(U-\bar{R}_{m}(X)\right) \cap\left(U-\bar{R}_{m}(Y)\right)$,

$$
\begin{aligned}
& =U-\left(\bar{R}_{m}(X) \cup \bar{R}_{m}(Y)\right) \subset U-\bar{R}_{m}(X \cap Y) \\
& =N E G_{m}(X \cap Y) . \square
\end{aligned}
$$

\section{Conclusions}

The objectives of this work are to study a new alternative method of data mining. It is about the rough set theory and its generalisations to topological notions used for the mining of decision rules. The advantage of these generalisations is a mathematical base of rough sets and the possibility of mathematical description of this problem. Rough sets seem to be advantageous for mining of incomplete information as well as for other algorithms.

\section{References}

Abd El-Monsef, M.E., El-Deeb, S.N. and Mahmoud, R.A. (1983) ' $\alpha$-open sets and $\alpha$-continuous mappings', Bull. Fac. Assiut. Uni., Vol. 12, No. 1, pp.77-90.

Abu-Donia, H.M. (2008) 'Comparison between different kinds of approximations by using a family of binary relations', Knowledge-Based Systems, Vol. 21, No. 8, pp.911-919.

Abu-Donia, H.M. and Salama, A.S. (2008) ' $\beta$-approximation spaces', Journal of Hybrid Computing Research, July-December, Vol. 1, No. 2, pp.67-83.

Abu-Donia, H.M., Nasef, A.A. and Marei, E.A. (2007) 'Finite information systems', Applied Mathematics and Information Sciences, Vol. 1, No. 1, pp.13-21.

Alpigini, J.J., Peters, F.J., Skowron, A. and Zhong, N. (2002) 'Rough set elements', 5th Int. Conf. on Rough Sets and Current Trends in Computing, Springer, Malvern, PA, USA, pp.12-16.

Banerjee, M. and Pal, S.K. (1995) 'Roughness of a fuzzy set', Information Sci., Vol. 93, Nos. 3-4, pp.235-246.

Biswas, R. (1992) 'On rough sets and fuzzy rough sets', Bull. Polish Acad. Sci. Math., Vol. 42, No. 1, pp.343-349.

Biswas, R. (1994) 'On rough fuzzy sets', Bull. Polish Acad. Sci. Math., Vol. 42, Nos. 2-3, pp.352-355.

Bonikowski, Z. (1994) 'Algebraic structures of rough sets', in Ziarko, W. (Ed.): Rough Sets, Fuzzy Sets and Knowledge Discovery, pp.243-247, Springer, London.

Bonikowski, Z., Bryniarski, E. and Wybraniec, U. (1998) 'Extensions and intentions in the rough set theory', Inform. Sci., Vol. 107, Nos. 1-4, pp.149-167.

Bryniaski, E. (1998) 'A calculus of rough sets of the first order', Bull. Polish Acad. Sci., Vol. 16, No. 1, pp.71-77.

Cattaneo, G. (1998) 'Abstract approximation spaces for rough theories', in Polkowski, L. and Skowron, A. (Eds.): Rough Sets in Knowledge Discovery 1: Methodology and Applications, pp.59-98, Physica-Verlag, Heidelberg.

Chakrabarty, K., Biswas, R. and Nanda, S. (2000) 'Fuzziness in rough sets', Fuzzy Sets and Systems, Vol. 110, No. 2, pp.247-251. 
Chen, X. and Li, Q. (2007) 'Construction of rough approximations in fuzzy setting', Fuzzy Sets and Systems, Vol. 158, No. 23, pp.2641-2653.

Chuchro, M. (1993) 'On rough sets in topological Boolean algebras', in Ziarko, W.P. (Ed.): Rough Sets, Fuzzy Sets and Knowledge Discovery, pp.157-166, Springer-Verlag, London.

Degang, C., Wenxia, Y. and Fachao, L. (2008) 'Measures of general fuzzy rough sets on a probabilistic space', Information Sciences, Vol. 178, No. 16, pp.3177-3187.

Deng, T., Chen, Y., Xu, W. and Dai, Q. (2007) 'A novel approach to fuzzy rough sets based on a fuzzy covering', Information Sciences, Vol. 177, No. 11, pp.2308-2326.

Dubois, D. and Prade, H. (1990) 'Rough fuzzy sets and fuzzy rough sets', Internat. J. Gen. Systems, Vol. 17, Nos. 2-3, pp.191-208.

Gong, Z., Sun, B. and Chen, D. (2008) 'Rough set theory for the interval-valued fuzzy information systems', Information Sciences, Vol. 178, No. 8, pp.1968-1985.

Hatir, E. and Noiri, T. (2006) 'Decompositions of continuity and complete continuity', Acta. Math. Hungary, Vol. 113, No. 4, pp.281-287.

Kryszkiewicz, M. (1998) 'Rough set approach to incomplete information systems', Inform. Sci., Vol. 112, Nos. 1-4, pp.39-49.

Lashin, E.F., Kozae, A.M., Abo Khadra, A.A. and Medhat, T. (2005) 'Rough set theory for topological spaces', International Journal of Approximate Reasoning, Vol. 40, Nos. 1-2, pp.35-43.

Levine, N. (1963) 'Semi open sets and semi continuity topological spaces', Amer. Math. Monthly, Vol. 70, No. 1, pp.24-32.

Li, T.J., Leung, Y. and Zhang, W.X. (2008) 'Generalized fuzzy rough approximation operators based on fuzzy coverings', International Journal of Approximate Reasoning, Vol. 48, No. 3, pp.836-856.

Liu, G. (2008a) 'Axiomatic systems for rough sets and fuzzy rough sets', International Journal of Approximate Reasoning, Vol. 48, No. 3, pp.857-867.

Liu, G. (2008b) 'Generalized rough sets over fuzzy lattices', Information Sciences, Vol. 178, No. 6, pp.1651-1662.

Liu, G. and Sai, Y. (2009) 'A comparison of two types of rough sets induced by coverings', International Journal of Approximate Reasoning, Vol. 50, No. 3, pp.521-528.

Nakamura, A. (1988) 'Fuzzy rough sets', Note on Multiple-valued Logic in Japan, Vol. 9, No. 2, pp.1-8.

Nanda, S. and Majumda, S. (1992) 'Fuzzy rough sets', Fuzzy Sets and Systems, Vol. 45, No. 2, pp.157-160.

Ouyang, Y., Wang, Z. and Zhang, H. (2010) 'On fuzzy rough sets based on tolerance relations', Information Sciences, Vol. 180, No. 4, pp.532-542.

Pawlak, Z. (1982) 'Rough sets', Int. J. of Computer and Information Sciences, Vol. 11, No. 5, pp.341-356. 